\title{
p62 manipulation affects chlorin e6-mediated photodynamic therapy efficacy in colorectal cancer cell lines
}

\author{
JU HEE KIM ${ }^{1}$ and IN-WOOK KIM ${ }^{2}$ \\ ${ }^{1}$ Biomedical Research Institute, Seoul National University Hospital, Seoul 03080; ${ }^{2}$ Department of Bioengineering, \\ College of Engineering, Hanyang University, Seoul 04763, Republic of Korea
}

Received October 28, 2019; Accepted February 24, 2020

DOI: $10.3892 / \mathrm{ol} .2020 .11522$

\begin{abstract}
. p62 is a multifunctional protein that mediates cell signaling pathways, autophagy and tumorigenesis, and participates in important regulation processes at the intersection between autophagy and cancer. Photodynamic therapy (PDT) is a treatment that involves photosensitizing agents and light to kill cancer cells. However, whether the efficacy of PDT depends on the expression level of p62 in colorectal cancer cell lines is not known. The present study aimed to examine the role of p62 expression levels in chlorin e6-based PDT in colorectal cancer cells. To study the effect of p62 on cancer cell death, we used PDT to treat a stable cell line overexpressing p62. Cells overexpressing p62 showed a higher cell death rate than cells not expressing this protein. Overexpression of p62 may contribute to colorectal cancer cell death. These results provide preliminary evidence for use of p62 as a therapy target to treat colorectal cancer.
\end{abstract}

\section{Introduction}

Colorectal cancer is the third most commonly diagnosed cancer and fourth leading cause of cancer-related deaths (1). Colorectal cancer is associated with a high risk of metastasis and recurrence despite an increased availability of diagnostic and therapeutic strategies. To treat patients with colorectal cancer, an approach that selectively targets cancer cells without damaging normal cells and which minimizes the risk of perforating the intestinal barrier is needed (2).

Photodynamic therapy (PDT) can be used to treat colorectal cancer. PDT is considered as a complementary therapy aimed at preventing tumor recurrence after surgical resection of colorectal cancer (3), making it a suitable approach for continuous removal of small fractions of tumors (4). PDT

Correspondence to: Professor In-Wook Kim, Department of Bioengineering, College of Engineering, Hanyang University, 222 Wangsimni-ro, Seongdong-gu, Seoul 04763, Republic of Korea E-mail: iwkim@hanyang.ac.kr

Key words: p62, colorectal cancer, therapeutic agents, apoptosis, tumor genesis, autophagy has been reported to be effective for treating aggressive colon cancer showing a high level of the vascular endothelial growth factor, which promotes tumor growth through angiogenesis (5). It has also been reported that PDT is an effective alternative treatment for drug-resistant colorectal cancer (6). PDT uses a modality-based photosensitizer, which selectively affects cancer cells, using excitation and light-absorption in the presence of oxygen to produce a high concentration of reactive oxygen species (ROS), such as singlet oxygen and other free radicals (7-9). The resulting damage cannot be overcome by the antioxidant system to protect the cell from oxidative damage, leading to necrosis, apoptosis, or autophagy of the target cell and tissue $(10,11)$. Chlorin e6 (Ce6) is a second-generation photosensitizer with strong absorption in the red spectrum and can be easily synthesized from chlorophyll. Irradiated $\mathrm{Ce} 6$ produced singlet oxygen which rapidly induces tumor cell death. Significant clinical benefits have been obtained with Ce6-mediated PDT (Ce6-PDT) in the treatment of various cancers including melanoma, bladder, and colorectal cancer (12).

Autophagy is a cellular pathway that removes damaged organelles and aggregates of lysosomal degradation to maintain the stability of the intracellular environment $(13,14)$. Autophagy contributes to the prolonged survival of tumor cells, whereas defects in autophagy play a critical role in tumorigenesis $(15,16)$. However, the exact function of autophagy in PDT for colorectal cancer treatment is unclear. A recent study showed that the hypoxic environment produced by PDT can induce autophagy in tumor cells (17). This suggests that autophagy is a form of adaptation of the nutritional environment of rapidly growing tumor cells in response to hypoxia. Oxidative stress has been shown to correlate with increasing protein toxicity, linking loss of protein, accumulation of ROS, and protein aggregate formation. Concurrently, p62 gene expression is increased by oxidative stress through activation of the transcription factor NF-E2-related factor 2 (18).

Furthermore, p62 is a multifunctional adapter protein involved in selective autophagy, cellular signaling pathways, and tumorigenesis $(19,20)$. Overexpression of p62 is likely related to tumorigenesis and has been observed in many types of tumors $(13,21-23)$, such as chemotherapy-resistant epithelial cell carcinoma (24). Analysis of autophagy-deficient mice showed that autophagy acts as a tumor suppressor by removing 
p62 (25). Moreover, abnormal expression of p62 was found in different types of cancer, suggesting a functional relationship between p62 accumulation and cancer progression (26). However, the role of p62 in the antitumor effects of PDT remains unclear.

This study was conducted to investigate the role of p62 in the antitumor effect of PDT for colorectal cancer. To understand whether p62 is directly related to PDT efficacy, we established a colorectal cancer cell line stably overexpressing p62 and tested PDT efficacy using an in vitro system. In vivo studies of p62-overexpressing cells were conducted to confirm the antitumor effects in xenograft mouse models. We found that PDT showed better effects in p62 overexpressing cells. Our findings suggest that p62 improves the antitumor efficacy of PDT.

\section{Materials and methods}

Materials. Chlorin e6 (Ce6) was obtained from Frontier Scientific, Inc.. Thiazolyl blue tetrazolium bromide (MTT) was from Sigma-Aldrich. Mouse monoclonal antibodies to p62 and $\beta$-actin were from Santa Cruz Biotechnology. 5-Fluorouracil (5-FU) and oxalitin were obtained from Sigma-Aldrich. Anti-p62 was obtained from Novusbio. Anti-HA was obtained from Santa Cruz Biotechnology. Anti-caspase 3 was obtained from Cell Signaling Technology.

p62 mRNA levels in colorectal cancer cell lines. After downloading RNA-sequencing data for 58 human colon colorectal cancer cell lines from the cancer cell line Encyclopedia (https://portals.broadinstitute.org/ccle), the relative fold-changes in p62 mRNA levels were categorized according to the classification of cells and graphically plotted.

Cell culture and in vitro photodynamic treatment. SW480, HCT116, LoVo, and DLD1 cells were maintained in atmosphere of $5 \% \mathrm{CO}_{2}$ in RPMI medium (Genedepot) supplemented with $10 \%$ fetal bovine serum and $1 \%$ penicillin-streptomycin solution. The cells were incubated with or without $\mathrm{Ce} 6$ $(0-400 \mathrm{nM})$ for $6 \mathrm{~h}$ at $37^{\circ} \mathrm{C}$. The cells were then photoirradiated using a diode laser-emitting red light at a wavelength of $670 \mathrm{~nm}$ (equipment from L2K Co., Ltd.). The power density at the illumination area was $800 \mathrm{~mW} / \mathrm{cm}^{2}$ and the total light dose was $4 \mathrm{~J} / \mathrm{cm}^{2}$. The cells were harvested at 4,8 , and $24 \mathrm{~h}$.

Cell viability assay. Cells were cultured overnight on 96-well culture plates $\left(1 \times 10^{4}\right.$ cells/well). Cells undergoing PDT were incubated for $4 \mathrm{~h}$ with $0.5 \mathrm{mg} / \mathrm{ml}$ thiazolyl blue tetrazolium bromide. Converted MTT formazan crystals were treated with dimethyl sulfoxide (Sigma). Absorbance at $540 \mathrm{~nm}$ was measured with a microplate reader.

Immunoblotting. Cells were lysed in buffer containing $20 \mathrm{mM}$ HEPES (pH 7.0), 1\% Triton X-100, 150 mM NaCl, 10\% glycerol, 2 mM EGTA, 1 mM EDTA, 1 mM glycerol 2-phosphate, $1 \mu \mathrm{g} / \mathrm{ml}$ leupeptin, $1 \mu \mathrm{g} / \mu \mathrm{l}$ aprotinin, $1 \mathrm{mM}$ AEBSF, $50 \mathrm{mM}$ $\mathrm{NaF}$, and $1 \mathrm{mM} \mathrm{Na} \mathrm{VO}_{4}$. The proteins, separated by SDS-PAGE, were transferred to a nitrocellulose membrane using an electrophoresis tank. After the membrane was incubated with specific antibodies, the signal was enhanced with chemiluminescence reagents (Genedepot) and then measured with a LAS-3000 (Fujifilm).

Stable cell establishment in colorectal cancer cells using retrovirus. To prepare SW480/p62, DLD1/p62, LoVo/p62, and HCT116/p62 cells, a retrovirus encoding HA-p62 genes was produced using the pMSCV-GFP vector. HEK293T cells were transfected with the pMSCV-GFP-HA-p62, pgag-pol, and pVSV-G, using Lipofectamine 2000 (Life Technologies). After $48 \mathrm{~h}$, the media containing retroviruses were collected and filtered to remove cell debris. Cells were infected and inoculated with the p62 retrovirus and then cells expressing p62 were selected with puromycin (Thermo Fisher Scientific).

Knockdown of p62 expression with shRNA and siRNA. To knock down p62 expression in colorectal cancer cell lines, the cells were transfected with negative control short interfering RNA (siRNA) or p62 siRNAs (Santa Cruz Biotechnology) using Lipofectamine 2000. Cells were also transfected with negative control short hairpin RNA (shRNA) or p62 shRNAs (Santa Cruz Biotechnology) using Lipofectamine 2000.

Immunocytochemical assay. Cells were cultured on a glass coverslip. Glass coverslips of confluent cells were washed twice with phosphate-buffered saline (PBS). Cells were fixed for $20 \mathrm{~min}$ at room temperature in $4 \%$ paraformaldehyde in PBS. After rinsing with PBS, cells were incubated with $2 \%$ FBS/PBS containing $0.05 \%$ Triton $\mathrm{X}-100$ for $30 \mathrm{~min}$ to block nonspecific staining. After washing with PBS, the cells were incubated overnight with an anti-HA antibody in $2 \%$ FBS/PBS, containing $0.05 \%$ Triton X-100 (1:200). After washing with PBS, the cells were incubated with secondary Alexa-647 antibody in $2 \%$ FBS/PBS containing $0.05 \%$ Triton X-100 for $1 \mathrm{~h}$. The cells were monitored by fluorescence microscopy (Axiovert 200 MAT, Zeiss).

Xenograft model and PDT. Four-week-old male BALB/c nude mice were used for the in vivo study. All facilities were approved by the Association for Assessment and Accreditation of Laboratory Animal Care. The present animal care and use protocol was reviewed and approved by the Institutional Animal Care and Use Committee (IACUC) in College of Medicine, Hanyang University of Korea (approval no. 2019-0043A). SW480/CTRL and LoVo/p62 cells $\left(0.5 \times 10^{6}\right)$ were inoculated subcutaneously in $100 \mu \mathrm{l}$ of PBS. The mice were then divided into treatment and control groups. The treatment group consisted of two subgroups: the control group and PDT group $(670 \mathrm{~nm})$.

Fluorometer analysis of intracellular photosensitizer levels. A total of $1 \times 10^{5}$ cells per well (2-ml cell suspension of SW480, HCT116, LoVo, and DLD1) were seeded in 6-well plates. The medium was then replaced with $2 \mathrm{ml}$ of fresh medium, containing $5 \mu \mathrm{M} \mathrm{Ce} 6$, and incubated for $1-24 \mathrm{~h}$. The solutions were removed, and the cells were rinsed with PBS $(1 \mathrm{ml})$. Afterward, the cells were harvested and centrifuged at 1,500 rpm for $5 \mathrm{~min}$. Pellets were subsequently washed with PBS $(1 \mathrm{ml})$ and centrifuged again. The fluorescence of the supernatants was measured with a Synergy MX fluorometer (BioTek) with excitation and emission wavelengths of 500 and 
A

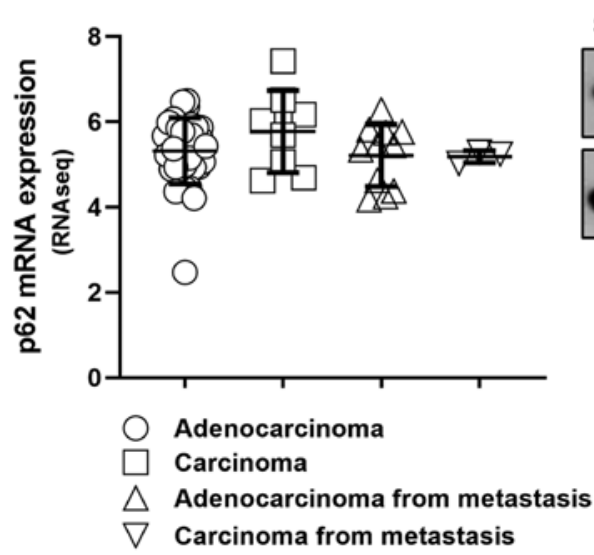

C
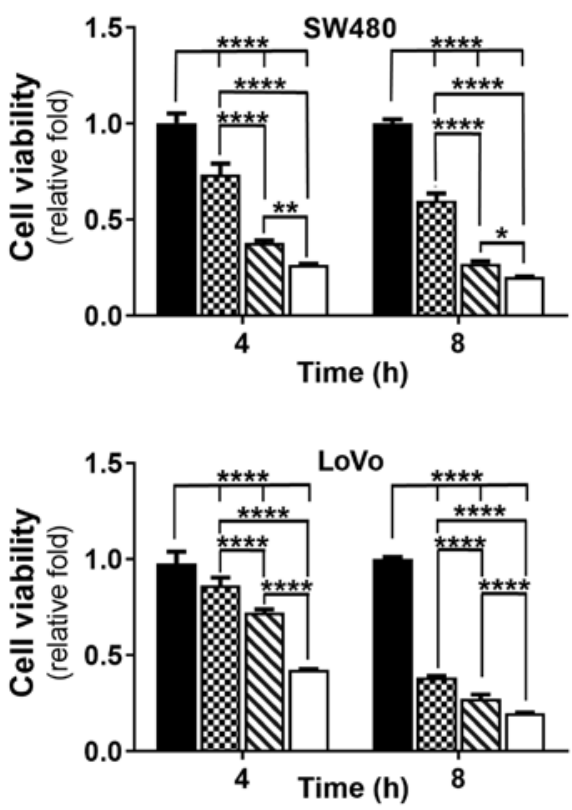

B

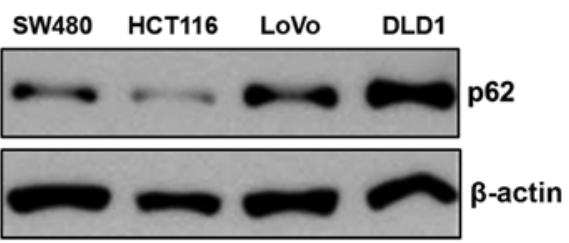

\section{$0 \mu \mathrm{M} \quad \mathrm{Q} 2.5 \mu \mathrm{M} \otimes 5 \mu \mathrm{M}$}

$10 \mu \mathrm{M}$

Figure 1. Differences in the PDT effect depending on the p62 expression level in colon cancer cell lines. (A) Relative fold-changes in p62 mRNA levels for 58 human colon colorectal cancer cell lines (Cancer Cell Line Encyclopedia datasets) were categorized according to the classification of cells. (B) Immunoblotting analysis of whole-cell lysates of various colon cancer cell lines. (C) Colon cancer cell lines were irradiated with a PDT laser $\left(4 \mathrm{~J} / \mathrm{cm}^{2}\right)$ after $6 \mathrm{~h}$ of pretreatment with $\mathrm{Ce} 6$ at the indicated concentrations. The MTT assay was performed in triplicate at $24 \mathrm{~h}$ following irradiation. Data are presented as the mean \pm SEM of three experiments. Data were analyzed with ANOVA and Tukey's post hoc test. ${ }^{*} \mathrm{P}<0.05,{ }^{* *} \mathrm{P}<0.01,{ }^{* * * * *} \mathrm{P}<0.0001$. PDT, photodynamic therapy.

$670 \mathrm{~nm}$, respectively. A calibration curve was then used to calculate the concentrations of Ce6 per 10,000 cells.

Statistical analysis. Data are presented as the mean \pm SEM. ANOVA and Turkey's post hoc test was used to compare the means between three or more groups. Student's t-test was performed to compare the means between two groups. $\mathrm{P}<0.05$ was considered to indicate a statistically significant difference. Statistical analysis was performed using GraphPad Prism 8 (GraphPad Software).

\section{Results}

Relationship between p62 and PDT treatment effects. We examined whether p62 protein enhances PDT efficacy in colorectal cancer. First, we checked the level of p62 expression in colorectal cancer cells using Cancer Cell Line Encyclopedia RNA sequencing data (27). The expression level of p62 was high in most colorectal cancer cells regardless of the distribution of adenocarcinoma, carcinoma, and metastatic cells (Fig. 1A). To determine whether there were differences in the expression of p62 according to the classification of colorectal cancer cells, we examined p62 protein expression in adenocarcinoma, SW480 and DLD1, carcinoma, HCT116, adenocarcinoma from metastasis, and LoVo cells. We checked the levels of p62 protein expression for each type of colorectal cancer. LoVo and DLD1 cells showed higher p62 levels compared to SW480 and HCT116 cells; there were no differences in the expression of p62 according to cell type (Fig. 1B). To measure the cellular uptake of photosensitizer, we indirectly examined the 
A
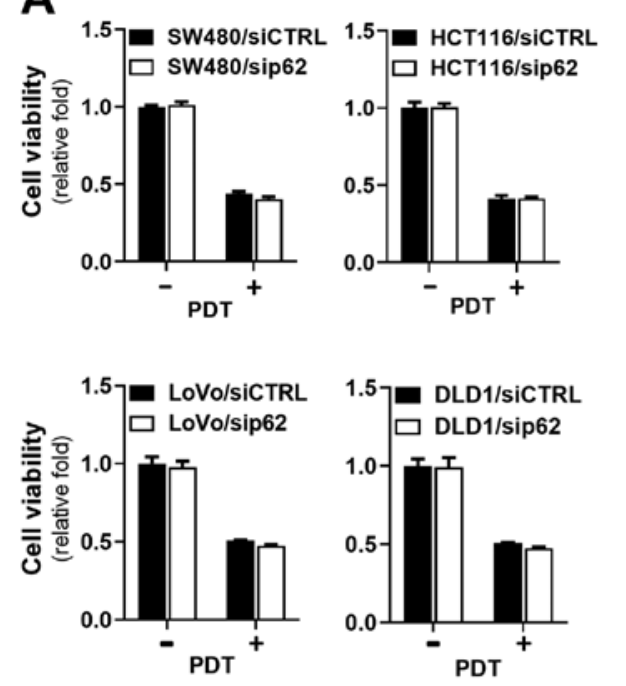

B



C
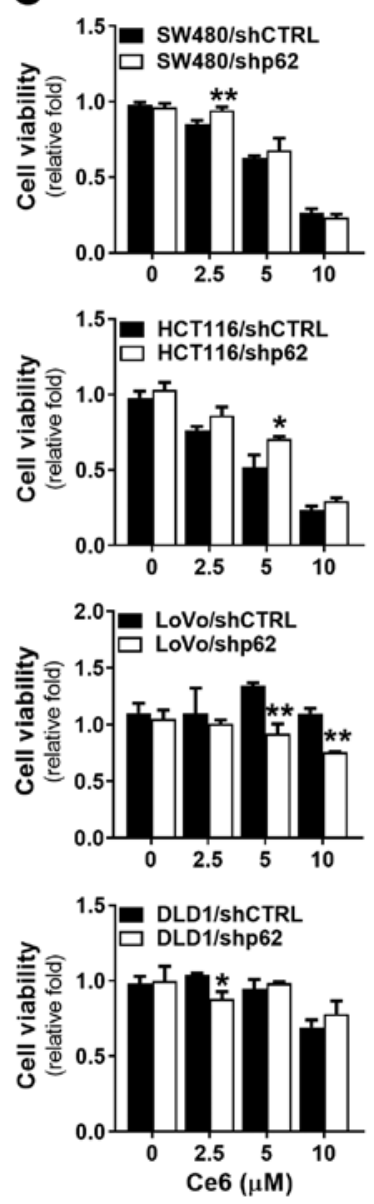

Dark toxicity
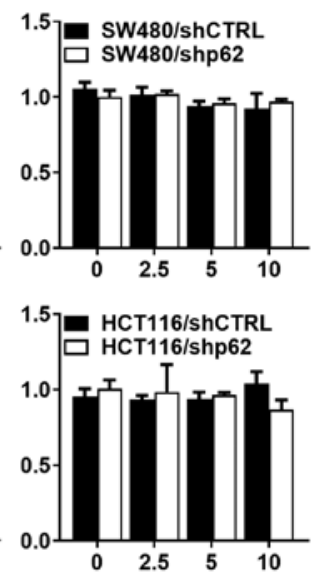

${ }^{1.5}[$ LoVo/shCTRL

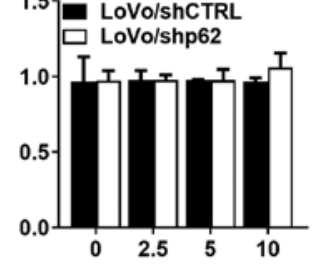

${ }^{1.5}$ DLD1/shCTRL



Figure 2. Effect of p62 deletion on colorectal cancer cell lines in PDT. (A) p62 siRNA-transfected cells were incubated with $100 \mathrm{nM}$ chlorin e6 for $6 \mathrm{~h}$ prior to irradiation. Cell viability was measured by an MTT assay. (B) Level of p62 in p62 shRNA-treated cells was determined by immunoblotting. (C) Cell viability was measured by an MTT assay after applying PDT to shRNA or p62 shRNA-treated cells. Data are presented as the mean \pm SEM of three experiments. ${ }^{*} \mathrm{P}<0.05,{ }^{* *} \mathrm{P}<0.01$ vs. respective shCTRL. si, small interfering; PDT, photodynamic therapy; sh, short hairpin; CTRL, control.

amount of Ce6 remaining in the cell by fluorometry analysis over time (Fig. S1). The amount of Ce6 remaining in the cell varied between the colorectal cancer cell types. SW480 cells showed a high residual amount of Ce6 until after 9 h. HCT116 cells exhibited the highest levels of $\mathrm{Ce} 6$ at $6 \mathrm{~h}$, and not much remained after that. LoVo cells showed high levels of residual $\mathrm{Ce} 6$ at all time points. Overall, the residual amount of $\mathrm{Ce} 6$ in DLD1 cells was similar to that in other cells, however, the amount remaining after $6 \mathrm{~h}$ was low. Therefore, PDT after $6 \mathrm{~h}$ incubation showed high intracellular uptake of $\mathrm{Ce} 6$ in most cells. To understand whether there were differences in PDT efficacy depending on p62 expression levels, we tested the effect of PDT on colorectal cancer cell lines. At $4 \mathrm{~h}$ after irradiation, PDT treatment effects differed depending on the p62 expression level. Cell lines with low p62 expression, SW480 and HCT116, showed decreased cell viability in time- and Ce6 concentration-dependent manners (Fig. 1C). Cells with high p62 expression, LoVo and DLD1, showed low therapeutic effects at $4 \mathrm{~h}$ after PDT application. There was no difference in PDT efficacy depending on the p62 expression level at $8 \mathrm{~h}$ after PDT in most cell lines.

Inhibition of p62 expression is not related to the PDT effect. The effects of PDT on p62 in colorectal cancer cells were variable. To understand the role of p62 in the effectiveness of PDT, the p62 gene was knocked down in the colorectal cancer cells. We inhibited p62 gene expression using p62 siRNA before subjecting the cells to PDT. The effect of p62 silencing in SW480, HCT116, LoVo, and DLD1 cells was assayed by immunoblotting with anti-p62 antibody (Fig. S2). There were no differences in the effects of PDT between control siRNA cells and p62 siRNA-treated colorectal cancer cells (Fig. 2A). To confirm this result, we further examined p62 knockdown cell lines using shRNA to knock down p62, which was verified by western blotting (Fig. 2B) before shp62 cells were subjected to PDT. The viability of SW480 and HCT116 cells was slightly decreased following p62 protein expression by shp62 (Fig. 2C). The effect of PDT on HCT116 cells was significantly reduced at $5 \mu \mathrm{M}$ Ce6. However, LoVo and DLD1 cells, which showed relatively high levels of p62 at baseline and which remained high after inhibition, showed higher susceptibility to PDT. Overall, when p62 was low at baseline and p62 expression was strongly inhibited, the effect of PDT was decreased, whereas when p62 was high at baseline and p62 expression was only slightly inhibited, the effect of PDT was increased, suggesting that the p62 expression level is an important factor determining PDT efficacy in colorectal cancer cells. 


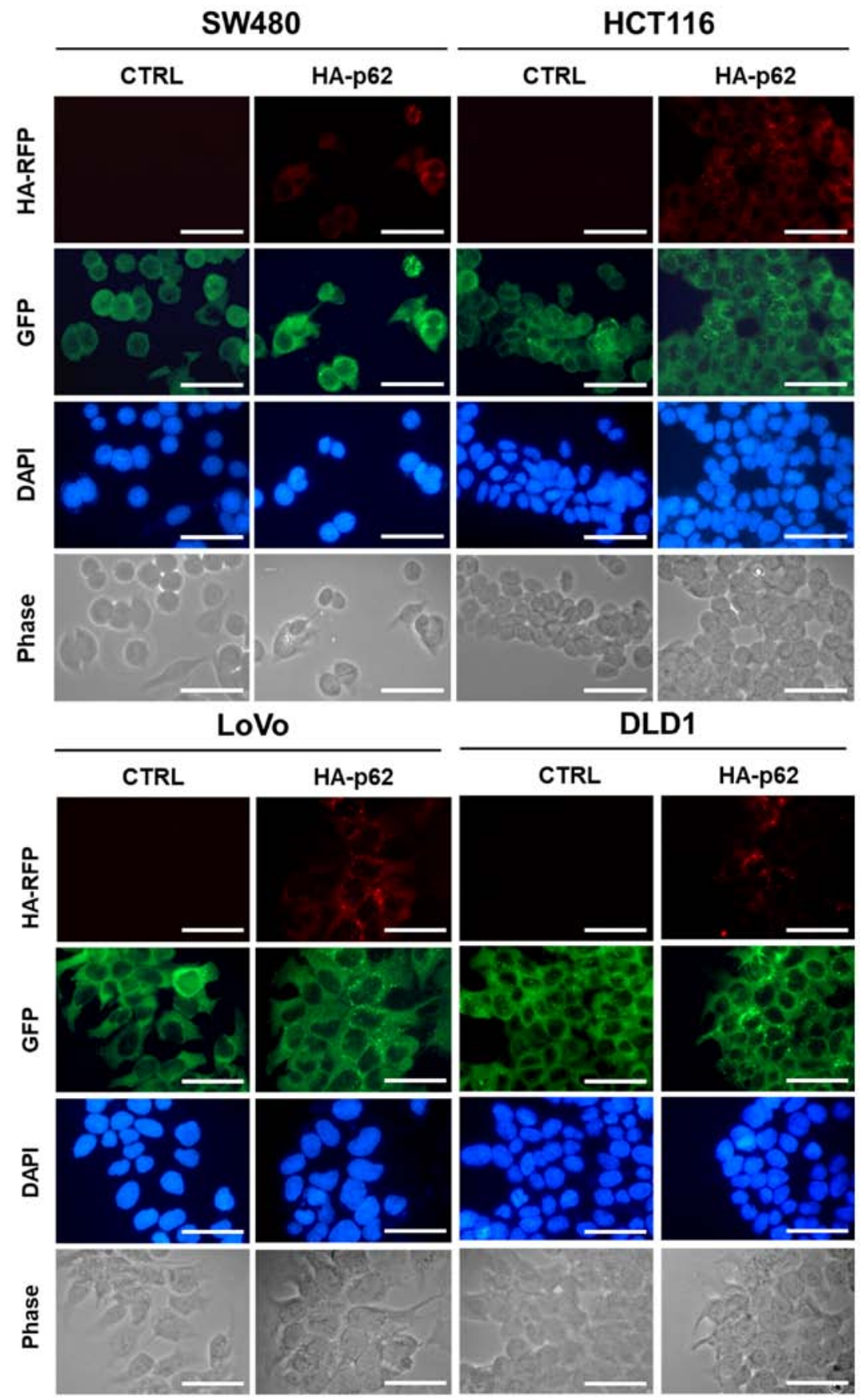

Figure 3. Establishment of p62 overexpressed colorectal cancer cell lines. Cells overexpressing p62 in colon cancer cell lines were confirmed by immunocytochemical assay using p62 and HA antibodies. Scale bar, $50 \mu \mathrm{m}$. CTRL, control.

p62 overexpression elevates PDT efficacy in colorectal cancer cells. We tested whether the p62 expression level affects PDT, as the data presented above suggested that inhibition of p62 expression had no effects on PDT of colorectal cancer cells. To confirm the intracellular expression level of p62, we also conducted immunocytochemistry analysis using anti-p62 antibody and anti-HA antibody to crosscheck the HA-tagged p62 expression level. Immunocytochemistry analysis showed that p62 was expressed mainly in the cytosol (Fig. 3). To confirm these observations, we overexpressed p62 in SW480, HCT116, LoVo, and DLD1 cells. The expression levels of p62 in SW480, HCT116, LoVo, and DLD1 cells were checked by immunoblot analysis along with anti-p62 antibody (Fig. 4A).
Next, we checked whether the PDT effect depended on p62 overexpression. Cells were incubated with Ce6 for $6 \mathrm{~h}$ and then irradiated with $4 \mathrm{~J} / \mathrm{cm}^{2}$ red light ( $670 \mathrm{~nm}$ wavelength), and then cell survival rate was measured after $6 \mathrm{~h}$ or $24 \mathrm{~h}$. There was no dark toxicity in which only $\mathrm{Ce} 6$ was treated without laser irradiation in all colorectal cancer cell lines (Fig. 4B). The cell lines showed relatively high expression level of p62. There was no difference in the PDT effect between the DLD1 and LoVo cell lines overexpressing p62 (Fig. 4B). In contrast, overexpression of p62 in colorectal cancer cells with normally low expression of p62 showed increased PDT effect (Fig. 4B). There was no significant difference in cell viability due to PDT between $6 \mathrm{~h}$ and $24 \mathrm{~h}$ after irradiation. To confirm the 


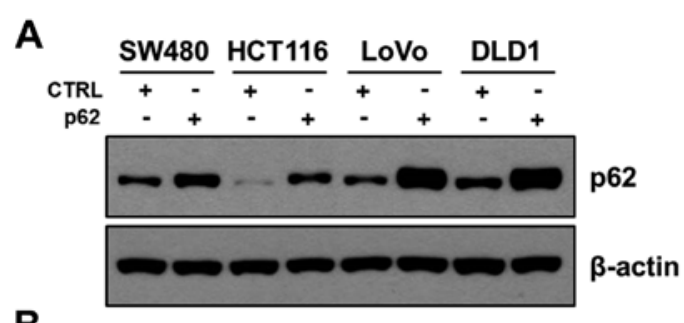

B

Phototoxicity (6 h) Phototoxicity (24 h) Dark toxicity (24 h)
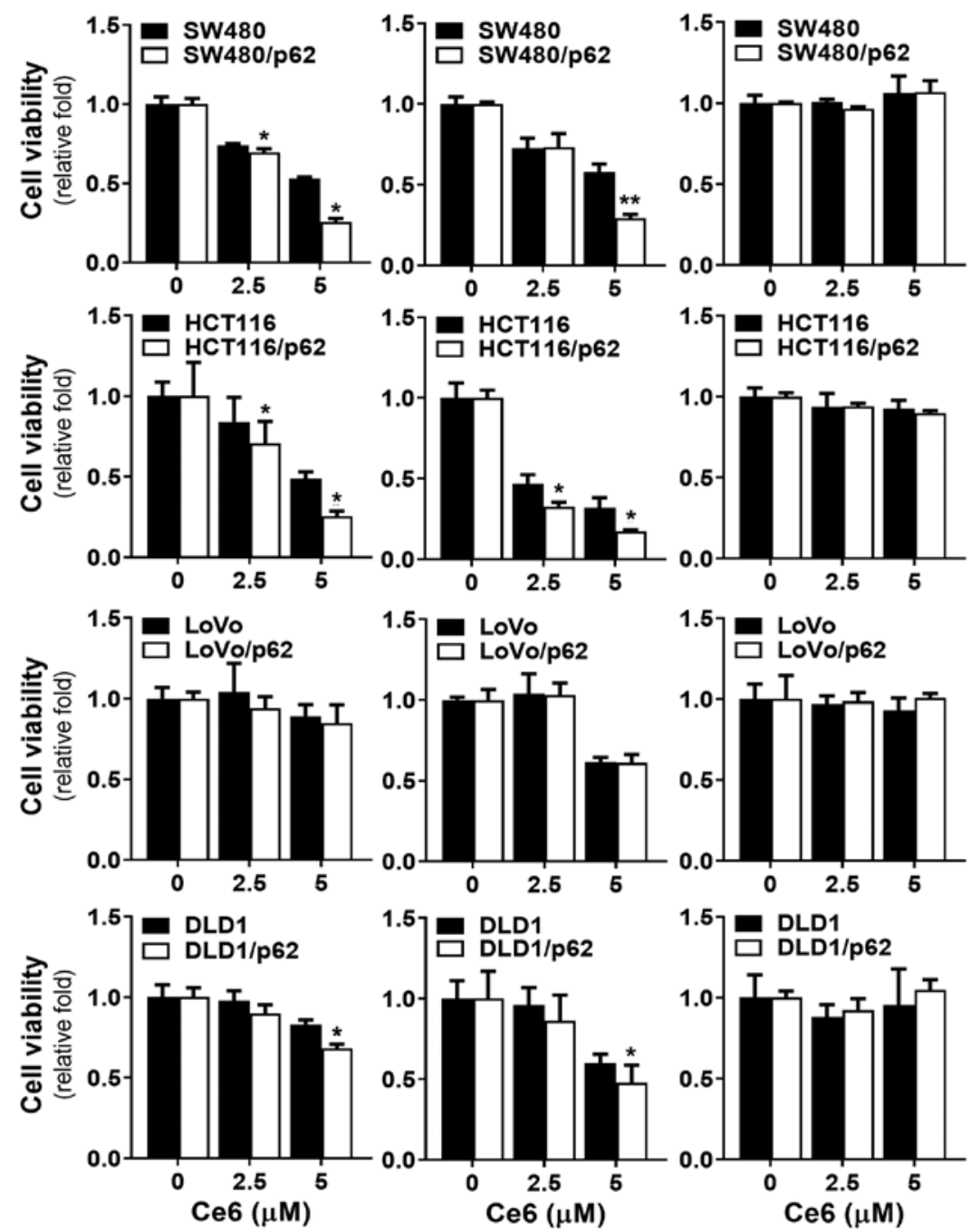

Figure 4. Effect of p62 overexpression on colorectal cancer cells. (A) Cells overexpressing p62 in colon cancer cell lines were confirmed by immunoblotting using a p62 antibody. (B) Cells were incubated with chlorin e 6 at the indicated concentrations for 6 h, before the MTT assay. Data are presented as the mean \pm SEM of three experiments. ${ }^{*} \mathrm{P}<0.05,{ }^{* *} \mathrm{P}<0.01$ vs. respective control cells.

effect of p62 on PDT efficacy, we evaluated cleaved caspase-3, a marker of apoptosis. SW480 and HCT116 cells with low p62 expression showed a higher rate of apoptosis compared to cells overexpressing p62 (Fig. 5A). In the MTT assay, LoVo cells showed the same PDT effect regardless of the level of p62 overexpression (Fig. 4B), and the apoptosis marker had no effect on p62 overexpression (Fig. 5B). These data suggest that p62 overexpression in colorectal cancer cell is be directly related to PDT efficacy, that p62 plays a role in inducing a higher rate of cancer cell death.

Variation of other drug effects by p62 overexpression. To confirm whether other therapeutic drugs were also affected by p62 overexpression, we tested oxalitin (Fig. 6A) and 5-FU (Fig. 6B), representative drugs used in colorectal cancer, on colorectal cancer cell lines overexpressing p62. When treated with conventional compounds, most tested cell lines showed no significantly increased toxicity associated with p62 overexpression compared to PDT. However, the effects of oxalitin and 5-FU were significantly different in the DLD1 and LoVo cell lines. This suggests that PDT acts on different cell death pathways and that p62 overexpression increases the effect of PDT, but not those of other drugs.

Antitumoreffect ofp62 overexpression in PDT. Totest the effects of p62 overexpression-dependent PDT on tumors in vivo, PDT 


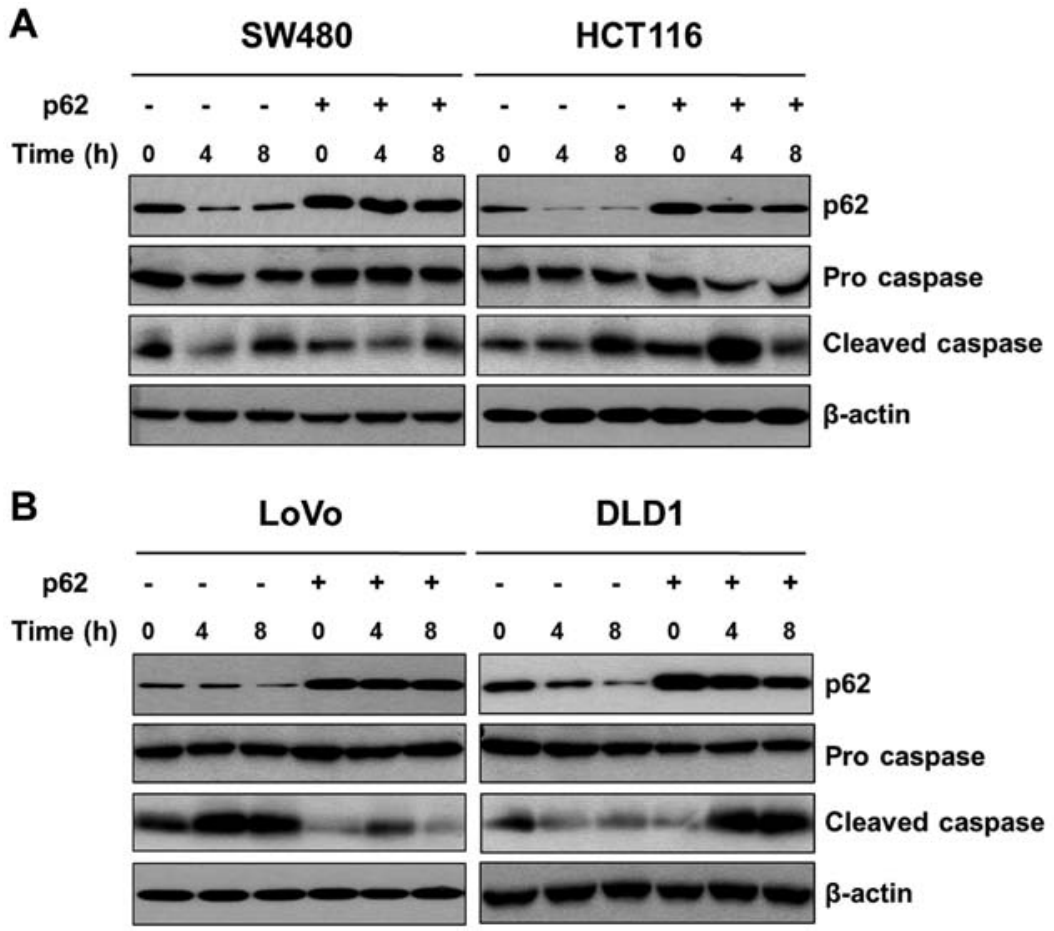

Figure 5. p62 overexpression affects apoptotic cell death. (A) SW480 and HCT116, and (B) LoVo and DLD1 cells were incubated with chlorin e6 at indicated concentrations for the indicated period, before immunoblotting using p62, caspase 3 and $\beta$-actin antibodies.

was applied to xenograft tumor models created by subcutaneous injection of colorectal cancer cells (SW480/SW480-p62, LoVo/LoVo-p62). When the tumor size reached $100-200 \mathrm{~mm}^{3}$, $1.25 \mathrm{mg} / \mathrm{kg}$ Ce 6 was administered to the tumors, and Ce6 was applied $6 \mathrm{~h}$ later followed by irradiation with a $670-\mathrm{nm}$ diode light $\left(150 \mathrm{~J} / \mathrm{cm}^{2}\right)$. There was no significant difference in tumorigenesis between SW480-p62 and SW480 cells. However, when PDT was applied, SW480-p62 cells showed a greater reduction in tumor size (Figs. 7A and S3). LoVo-injected tumors showed no difference between p62 overexpression and tumorigenesis. After PDT, the tumor size of LoVo-p62 cells was greater than that of LoVo cells. However, the reduction rate was lower than that of SW480 (Figs. 7B and S3), suggesting that the level of p62 overexpression affects PDT in vivo.

\section{Discussion}

We investigated whether elevated p62 expression significantly affects PDT efficacy. We also found that other chemotherapy treatments showed increased efficacy in cell lines overexpressing $\mathrm{p} 62$.

Previous studies suggested that $\mathrm{p} 62$ plays a role in tumor progression, with abnormal accumulation of p62 increasing the rate of tumorigenesis (26). Moreover, p62 has been identified as an effective substrate and regulator of autophagy (20). In PDT, simultaneously produced ROS induces autophagy and an apoptosis pathways in cancer cells (8). Combination therapy that includes an autophagy inhibitor increases the anticancer effect of PDT (28). p62 overexpression increased the therapeutic effect of PDT in in vitro and in vivo models. Furthermore, p62-knockdown cells, created by using shRNA, were slightly less susceptible to PDT. These data demonstrate that sufficient p62 expression is associated with the antitumor effect of PDT. Some previous studies reported that p62-deficient cells showed reduced aggregate formation with attenuated ROS levels, reduced apoptosis, and improved survival after PDT $(29,30)$. In tumor promotion, p62 has been associated with cancer therapy resistance, particularly, resistance to platinum-typed chemotherapy reagents (31). However, the p62 expression level is related to cisplatin-resistance, and insufficient p62 degradation leads to resistance to cell death in ovarian carcinoma (26). Most studies reported that autophagy increases tumor survival. PDT studies with porphyrin IX in HCT116 colon cancer cells reported that inhibition of autophagy was effective for PDT antitumor effects (32). In a study of sinoporphyrin sodium mediated PDT and photosan-II mediated PDT applied to human colorectal cancer cells, autophagy inhibition increased the efficacy of PDT $(33,34)$. In our study, overexpression of p62, which plays an important role in autophagy, increased the effect of PDT, conflicting with previously reported studies. However, it is unclear how p62 is associated with autophagy in the tumor environment caused by PDT. As we observed early effects after PDT, it is difficult to explain the association with autophagy, which is a limitation of this study and requires further analysis.

This study showed that the antitumor effect of platinum-based colorectal cancer chemotherapy with oxalitin and chemotherapy with 5-FU was improved when the expression of p62 was increased. PDT affects cancer cells by generating excess ROS, which is performed by a photosensitizer (29). In all cancer treatment methods, cell resistance limits cancer therapy efficacy and effectiveness. Resistance in PDT consists of inducing drug efflux on the cell surface and creating an internal resistance system (35).

Overall, p62 is an important substrate for autophagy and plays a role in tumor-genesis by maintaining homeostasis of 
A
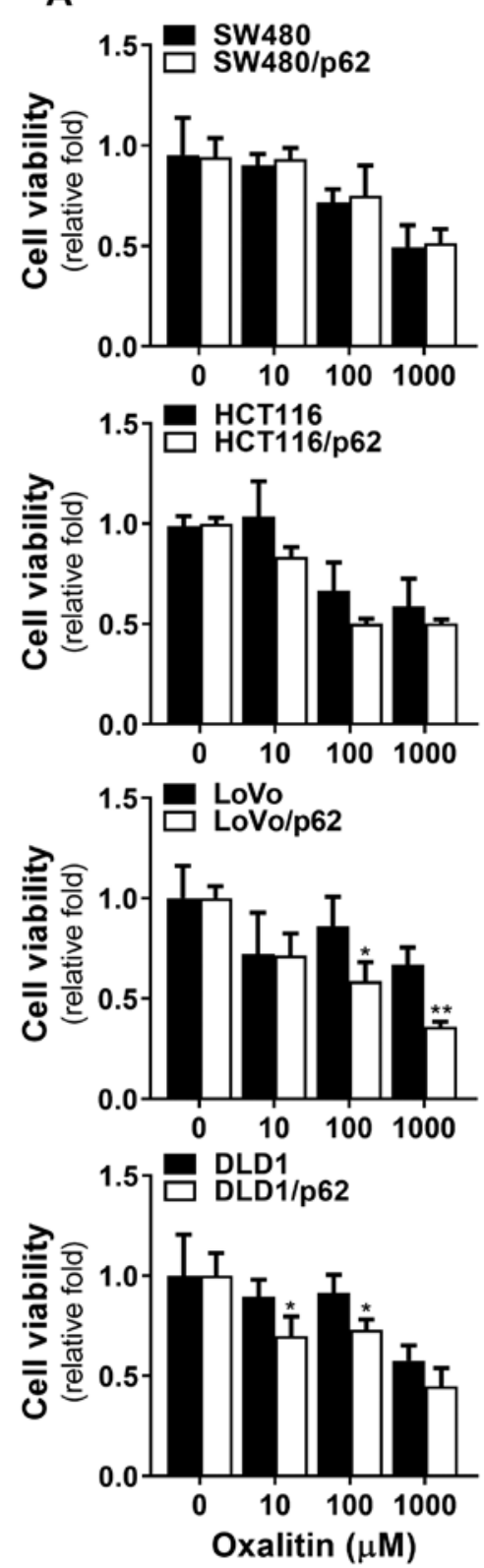

B


Figure 6. Differences in effect of oxalitin and 5-FU on p62 overexpression. (A) Cell viability was measured by an MTT assay after treatment with the indicated concentrations of oxalitin for $24 \mathrm{~h}$. (B) Cell viability was measured by an MTT assay after treatment with the indicated concentrations of 5-FU for $24 \mathrm{~h}$. Data are presented as the mean \pm SEM of three experiments. ${ }^{*} \mathrm{P}<0.05,{ }^{* *} \mathrm{P}<0.01$ vs. respective control cells. 5-FU, 5-fluorouracil.
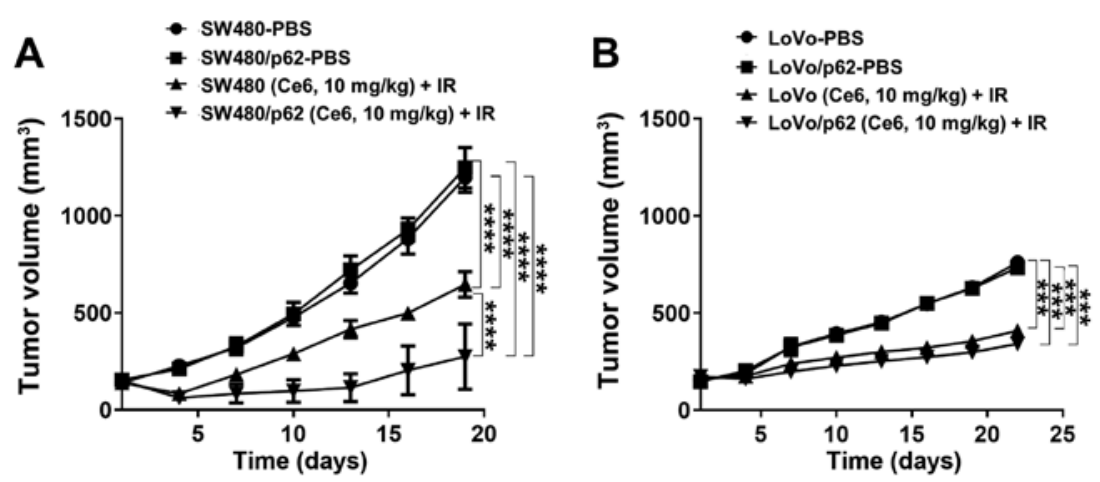

Figure 7. Antitumor effect of p62 overexpression in photodynamic therapy. (A) SW480 and SW480-p62 cells were collected and inoculated into BALB/c nude mice by subcutaneous injection. (B) LoVo and LoVo-p62 cells collected and inoculated into BALB/c nude mice by subcutaneous injection. Ce6 was administered to the tumors at a dose of $1.25 \mathrm{mg} / \mathrm{kg}$, and Ce 6 was applied $6 \mathrm{~h}$ later, followed by irradiation with a $670 \mathrm{~nm}$ diode light $\left(150 \mathrm{~J} / \mathrm{cm}^{2}\right)$. Tumor sizes were measured using a caliper, tumor volume was calculated using the formula: $0.523 \mathrm{x}$ length $\mathrm{x} \mathrm{width}^{2}\left(\mathrm{~mm}^{3}\right)$. Data are presented as the mean $\pm \mathrm{SEM}$. $\mathrm{n}=4$. Data were analyzed with ANOVA and Tukey's post hoc test. ${ }^{* * *} \mathrm{P}<0.001,{ }^{* * * * *} \mathrm{P}<0.0001$. Ce6, chlorin e6; IR, irradiation. 
the cancer cell microenvironment (36). We predicted that low expression of p62 would increase PDT efficacy. However, the results indicated that overexpression of p62 increased the effect of PDT. In most studies, inhibition of p62 has been reported to enhance antitumor effects; however, our findings suggest that over-expression of p62 would be more effective. Moreover, deletion of p62 in some colorectal cancer cells reduced their PDT susceptibility. Further studies of the effect of p62 expression on antitumor mechanisms are needed. Our results suggest that p62 is an important substance in antitumor effect processes and is a suitable candidate as a therapeutic target.

\section{Acknowledgements}

Not applicable.

\section{Funding}

The present study was supported by The Basic Science Research Program through The National Research Foundation of Korea (NRF) funded by The Ministry of Education, Science and Technology (grant nos. NRF-2017R1A2B4011122 and NRF-2019R1C1C1006898).

\section{Availability of data and materials}

All datasets generated and analyzed in the present study are included in this published article.

\section{Authors' contributions}

JHK and IWK conceived and designed the study, and wrote the manuscript JHK and IWK performed the experiments. JHK collected and analyzed the data. IWK interpreted the data and reviewed the manuscript. Both authors have read and approved the final version of this manuscript.

\section{Ethics approval and consent to participate}

The present animal care and use protocol was reviewed and approved by the Institutional Animal Care and Use Committee (IACUC) in College of Medicine, Hanyang University of Korea (approval no. 2019-0043A).

\section{Patient consent for publication}

Not applicable.

\section{Competing interests}

The authors declare that they have no competing interests.

\section{References}

1. Mármol I, Sánchez-de-Diego C, Pradilla Dieste A, Cerrada E and Rodriguez Yoldi MJ: Colorectal Carcinoma: A General Overview and Future Perspectives in Colorectal Cancer. Int J Mol Sci 18: 18, 2017.

2. Kawczyk-Krupka A, Bugaj AM, Latos W, Zaremba K, Wawrzyniec K and Sieron A: Photodynamic therapy in colorectal cancer treatment: The state of the art in clinical trials. Photodiagn Photodyn Ther 12: 545-553, 2015.
3. Shishkova N, Kuznetsova O and Berezov T: Photodynamic therapy in gastroenterology. J Gastrointest Cancer 44: 251-259, 2013.

4. Barr H, MacRobert AJ, Tralau CJ, Boulos PB and Bown SG: The significance of the nature of the photosensitizer for photodynamic therapy: Quantitative and biological studies in the colon. Br J Cancer 62: 730-735, 1990.

5. Kawczyk-Krupka A, Kwiatek B, Czuba ZP, Mertas A, Latos W, Verwanger T, Krammer B and Sieron A: Secretion of the angiogenic factor VEGF after photodynamic therapy with ALA under hypoxia-like conditions in colon cancer cells. Photodiagn Photodyn Ther 21: 16-18, 2018.

6. Halaburková A, Jendželovský R, Koval' J, Herceg Z, Fedoročko P and Ghantous A: Histone deacetylase inhibitors potentiate photodynamic therapy in colon cancer cells marked by chromatin-mediated epigenetic regulation of CDKN1A. Clin Epigenetics 9: 62, 2017.

7. Dolmans DE, Fukumura D and Jain RK: Photodynamic therapy for cancer. Nat Rev Cancer 3: 380-387, 2003.

8. Castano AP, Mroz P and Hamblin MR: Photodynamic therapy and anti-tumour immunity. Nat Rev Cancer 6: 535-545, 2006.

9. Kwiatkowski S, Knap B, Przystupski D, Saczko J, Kędzierska E, Knap-Czop K, Kotlińska J, Michel O, Kotowski K and Kulbacka J: Photodynamic therapy - mechanisms, photosensitizers and combinations. Biomed Pharmacother 106: 1098-1107, 2018.

10. Mroz P, Yaroslavsky A, Kharkwal GB and Hamblin MR: Cell death pathways in photodynamic therapy of cancer. Cancers (Basel) 3: 2516-2539, 2011.

11. Broekgaarden M, Weijer R, van Gulik TM, Hamblin MR and Heger M: Tumor cell survival pathways activated by photodynamic therapy: A molecular basis for pharmacological inhibition strategies. Cancer Metastasis Rev 34: 643-690, 2015.

12. Orenstein A, Kostenich G, Roitman L, Shechtman Y, Kopolovic Y, Ehrenberg B and Malik Z: A comparative study of tissue distribution and photodynamic therapy selectivity of chlorin e6, Photofrin II and ALA-induced protoporphyrin IX in a colon carcinoma model. Br J Cancer 73: 937-944, 1996.

13. Levy JMM, Towers CG and Thorburn A: Targeting autophagy in cancer. Nat Rev Cancer 17: 528-542, 2017.

14. Mathew R, Karantza-Wadsworth V and White E: Role of autophagy in cancer. Nat Rev Cancer 7: 961-967, 2007.

15. Amaravadi R, Kimmelman AC and White E: Recent insights into the function of autophagy in cancer. Genes Dev 30: 1913-1930, 2016.

16. Degenhardt K, Mathew R, Beaudoin B, Bray K, Anderson D, Chen G, Mukherjee C, Shi Y, Gélinas C, Fan Y, et al: Autophagy promotes tumor cell survival and restricts necrosis, inflammation, and tumorigenesis. Cancer Cell 10: 51-64, 2006.

17. Rodríguez ME, Catrinacio C, Ropolo A, Rivarola VA and Vaccaro MI: A novel HIF-1 $\alpha /$ VMP1-autophagic pathway induces resistance to photodynamic therapy in colon cancer cells. Photochem Photobiol Sci 16: 1631-1642, 2017.

18. Jain A, Lamark T, Sjøttem E, Larsen KB, Awuh JA, Øvervatn A McMahon M, Hayes JD and Johansen T: p62/SQSTM1 is a target gene for transcription factor NRF2 and creates a positive feedback loop by inducing antioxidant response element-driven gene transcription. J Biol Chem 285: 22576-22591, 2010.

19. Liu WJ, Ye L, Huang WF, Guo LJ, Xu ZG, Wu HL, Yang C and Liu HF: p62 links the autophagy pathway and the ubiqutin-proteasome system upon ubiquitinated protein degradation. Cell Mol Biol Lett 21: 29, 2016.

20. Rusten TE and Stenmark H: p62, an autophagy hero or culprit? Nat Cell Biol 12: 207-209, 2010.

21. Moscat J and Diaz-Meco MT: p62 at the crossroads of autophagy, apoptosis, and cancer. Cell 137: 1001-1004, 2009.

22. Islam MA, Sooro MA and Zhang P: Autophagic Regulation of p62 is Critical for Cancer Therapy. Int J Mol Sci 19: 19, 2018

23. Li SS, Xu LZ, Zhou W, Yao S, Wang CL, Xia JL, Wang HF, Kamran M, Xue XY, Dong L, et al: p62/SQSTM1 interacts with vimentin to enhance breast cancer metastasis. Carcinogenesis 38: 1092-1103, 2017.

24. Battista RA, Resnati M, Facchi C, Ruggieri E, Cremasco F, Paradiso F, Orfanelli U, Giordano L, Bussi M, Cenci S, et al: Autophagy mediates epithelial cancer chemoresistance by reducing p62/SQSTM1 accumulation. PLoS One 13: e0201621, 2018.

25. Kuma A, Komatsu M and Mizushima N: Autophagy-monitoring and autophagy-deficient mice. Autophagy 13: 1619-1628, 2017. 
26. Iwadate R, Inoue J, Tsuda H, Takano M, Furuya K, Hirasawa A Aoki D and Inazawa J: High Expression of SQSTM1/p62 Protein Is Associated with Poor Prognosis in Epithelial Ovarian Cancer. Acta Histochem Cytochem 47: 295-301, 2014.

27. Barretina J, Caponigro G, Stransky N, Venkatesan K, Margolin AA, Kim S, Wilson CJ, Lehár J, Kryukov GV, Sonkin D, et al: The Cancer Cell Line Encyclopedia enables predictive modelling of anticancer drug sensitivity. Nature 483 603-607, 2012.

28. Wei MF, Chen MW, Chen KC, Lou PJ, Lin SY, Hung SC, Hsiao M, Yao CJ and Shieh MJ: Autophagy promotes resistance to photodynamic therapy-induced apoptosis selectively in colorectal cancer stem-like cells. Autophagy 10: 1179-1192, 2014.

29. Agostinis P, Berg K, Cengel KA, Foster TH, Girotti AW, Gollnick SO, Hahn SM, Hamblin MR, Juzeniene A, Kessel D, et al: Photodynamic therapy of cancer: An update. CA Cancer J Clin 61: 250-281, 2011.

30. Rubio N, Verrax J, Dewaele M, Verfaillie T, Johansen T, Piette J and Agostinis P: p38(MAPK)-regulated induction of p62 and NBR1 after photodynamic therapy promotes autophagic clearance of ubiquitin aggregates and reduces reactive oxygen species levels by supporting Nrf2-antioxidant signaling. Free Radic Biol Med 67: 292-303, 2014

31. Yan XY, Zhang Y, Zhang JJ, Zhang LC, Liu YN, Wu Y, Xue YN, Lu SY, Su J and Sun LK: p62/SQSTM1 as an oncotarget mediates cisplatin resistance through activating RIP1-NF- $\kappa B$ pathway in human ovarian cancer cells. Cancer Sci 108: 1405-1413, 2017.

32. Ouyang G, Xiong L, Liu Z, Lam B, Bui B, Ma L, Chen X, Zhou P, Wang K, Zhang Z, et al: Inhibition of autophagy potentiates the apoptosis-inducing effects of photodynamic therapy on human colon cancer cells. Photodiagn Photodyn Ther 21: 396-403, 2018
33. Xiong L, Liu Z, Ouyang G, Lin L, Huang H, Kang H, Chen W, Miao X and Wen Y: Autophagy inhibition enhances photocytotoxicity of Photosan-II in human colorectal cancer cells. Oncotarget 8: 6419-6432, 2017.

34. Zhu B, Li S, Yu L, Hu W, Sheng D, Hou J, Zhao N, Hou X, Wu Y, Han Z, et al: Inhibition of Autophagy with Chloroquine Enhanced Sinoporphyrin Sodium Mediated Photodynamic Therapy-induced Apoptosis in Human Colorectal Cancer Cells. Int J Biol Sci 15: 12-23, 2019.

35. Olsen CE, Weyergang A, Edwards VT, Berg K, Brech A, Weisheit S, Høgset A and Selbo PK: Development of resistance to photodynamic therapy (PDT) in human breast cancer cells is photosensitizer-dependent: Possible mechanisms and approaches for overcoming PDT-resistance. Biochem Pharmacol 144: 63-77, 2017.

36. Reina-Campos M, Shelton PM, Diaz-Meco MT and Moscat J: Metabolic reprogramming of the tumor microenvironment by p62 and its partners. Biochim Biophys Acta Rev Cancer 1870: 88-95, 2018.

This work is licensed under a Creative Commons Attribution-NonCommercial-NoDerivatives 4.0 International (CC BY-NC-ND 4.0) License. 